\title{
Path Separability of Graphs
}

\author{
Emilie Diot and Cyril Gavoille \\ Université de Bordeaux, LaBRI, France \\ \{diot, gavoille\}@labri.fr
}

\begin{abstract}
In this paper we investigate the structural properties of $k$ path separable graphs, that are the graphs that can be separated by a set of $k$ shortest paths. We identify several graph families having such path separability, and we show that this property is closed under minor taking. In particular we establish a list of forbidden minors for 1-path separable graphs.
\end{abstract}

\section{Introduction}

"Divide and Conquer" is an common technique in computer science to solve problems. The whole data is separated into different small parts to find a solution in these parts, and then to merge the solutions to obtain the result on the input graph.

A wide theory has been developed for graphs that can be decomposed into small pieces. Such graphs, a.k.a. bounded treewidth graphs, supports polynomial algorithms for many class of problems, whereas no algorithms of complexity better than exponential complexity are known for the general case. This has contributed to new insights into Fixed Parameterized Tractable theory whose consequences for practical algorithms are effective improvements on the running time 26 .

There are however problems that can be efficient solved even for graphs without small separators (or equivalently of large treewidth). Large separators but "well shaped" reveal very useful for approximation algorithms. For instance, if the separator has a small diameter, or a small dominating set, then distances between vertices can be computed efficiently up to some small additive errors (see 4,3]12] for works about the treelength of a graph).

An important observation due to Thorup [11] is that separators consisting of a set of shortest paths are also very useful for the design of compact routing scheme, distance and reachability oracles. More precisely, he used the fact that every weighted planar graph with $n$ vertices has a set of three shortest paths whose deletion split the graph into connected components of at most $n / 2$ vertices (a decomposition into components of at most $2 n / 3$ vertices using two shortest paths was early proved in [7]). Using a recursive decomposition, and sampling

\footnotetext{
* The second author is also member of the "Institut Universitaire de France". Both authors are supported by the ANR-project "ALADDIN", and the équipe-projet commune LaBRI-INRIA Bordeaux Sud-Ouest "CÉPAGE".
} 
each such shortest path, he showed that distances between any pair of vertices can be approximated up to a factor of $1+\varepsilon$ in polylogarithmic time, for every $\varepsilon>0$, with an oracle of size $\left.11 \varepsilon^{-1} n \log n\right)$.

This notion of "shortest path" separator has been extended in 11. Roughly speaking, a $k$-path separator is the union of $k$ shortest paths whose removal halve the graph. The formal definition is actually slightly more complicated and is described in Section 2. The same authors have showed that $k$-path separable graphs have efficient solutions for several "Object Location Problems" including compact routing schemes, distance oracles, and small-world navigability. Based on the deep Robserton-Seymour's decomposition [9], they show in particular that every weighted graph excluding a minor $H$ has a $(1+\varepsilon)$-approximate distance oracle of size $O\left(\varepsilon^{-1} k n \log n\right)$, where $k=k(H)$ depending only of $H$. Actually, the oracle can be distributed into balanced labels, each of size $O\left(\varepsilon^{-1} k \log n\right)$ such that distance queries can be answered from given the source-destination labels only. The graphs excluding a fixed minor is a huge family of graphs including (and not restricted to) bounded treewidth graphs, planar graphs, and graphs of bounded genus.

\subsection{Our Results}

An approximate distance oracle for a graph $G$ is a data-structure that quickly returns, for any source-destination pair, an approximation on cost of a shortest path connecting them. Such data-structures are obtained by preprocessing $G$ where each edge has a weight corresponding to the cost of traversing this edge (or length). However, in practice, the number $n$ of vertices of $G$ is large whereas the number of interesting vertices for which we want approximate the distance is small (say $t$ ). Current solutions [111] provide oracles of size $O(t \log n)$ whereas a space independent of $n$ would be preferable.

Such a compression can be achieved by adding weights on the vertices of the input graph. Typically, interesting vertices receive a weight 1 whereas the others receive a weight 0 . A $k$-path separator on such vertex- and edge-weighted graph is then defined as previously, excepted that the removal of the separator must leave connected components of size at most half the total vertex-weight of the graph. The size of the oracles is improved since $\log t$ recursion levels suffice instead of $\log n$ in the initial formulation.

In this paper we extend the classical notion of $k$-path separability to edgeand vertex-weighted graphs. In particular, we prove that previous results (e.g., the 3-path separability of planar graphs) still hold in this new framework.

We establish a connection between separators corresponding to the border of a face and $k$-path separability, and we identify several families of graphs that are 1-path and 2-path separable. We note that most of our proofs are constructive, and lead to polynomial and even linear algorithms.

More interestingly, we show that the family of graphs that are $k$-path separable for any weight function is minor-closed. Combined with the Graph Minor Theory

${ }^{1}$ The size is actually the number of "distance items" stored in the oracle. 
of Robertson and Seymour [10, it follows that the $k$-path separability can be theoretically tested in cubic time [8] for each fixed $k$, although no algorithm is currently known. Finally, we provide a first step towards the characterization of 1-path separable graphs.

\section{Preliminaries}

A minor of a graph $G$ is a subgraph of a graph obtained from $G$ by edge contraction. We denote by $K_{r}$ the complete graph (or clique) on $r$ vertices, and $K_{p, q}$ the complete bipartite graph. For convenience, the term component is a short for connected component.

A vertex-weight function (resp. edge-weight function) is a non-negative real function defined on the vertices (resp. edges) of a graph. A non-negative real function applying on both vertices and edges is simply called weight function. A weighted graph is graph $G$ having a weight function $\omega$, that we denote also by $(G, \omega)$. The weight of a subgraph $H$ of $G$, denoted by $\omega(H)$, is the sum of the weights over the vertices of $H$.

A half-separator for a graph $G$ with vertex-weight function $\omega$ is a subset of vertices $S$ such that each component of $G \backslash S$ has weight at most $\omega(G) / 2$. Observe that the deletion of a half-separator does not necessarily disconnect the graph.

A $k$-path separator of a weighted graph $G$ is a subgraph $P_{0} \cup P_{1} \cup \ldots$ where each $P_{i}$ is a subgraph composed of the union of $k_{i}$ minimum cost paths in $G \backslash \bigcup_{j<i} P_{j}$, and where $\sum_{i} k_{i} \leqslant k$. A $k$-path separator is said strong if it consists of $P_{0}$ only, i.e., composed of the union of $k$ minimum cost paths in $G$. A weighted graph is (strongly) $k$-path separable if every induced subgraph has a (strong) $k$-path separator.

A tree-decomposition of a graph $G$ is a tree $T$ whose vertices, called bags, are subsets of vertices of $G$ such that:

1. for every vertex $u$ of $G$, there exists a bag $X$ of $T$ such that $u \in X$;

2. for every edge $\{u, v\}$ of $G$, there exists a bag $X$ of $T$ such that $u, v \in X$; and

3. for every vertex $u$ of $G$, the set of bags containing $u$ induces a subtree of $T$.

An important property following from the last two points is that every path between $u \in X$ and $v \in Y$ in $G$ has to intersect all the bags on the path from $X$ and $Y$ in $T$. Therefore, the deletion of every bag $X$ disconnects $G$ provided that $T \backslash X$ is composed of more than one subtree and that no bags $Y \subseteq X$.

The width of a tree-decomposition $T$ is $\max _{X \in T}\{|X|-1\}$. A treewidth- $t$ graph is a graph having a tree-decomposition of width $t$, and the treewidth of $G$ is the minimum $t$ such that $G$ is a treewidth- $t$ graph.

We will use several times the following basic result (the proof of this lemma, and of several others, appear in the full version).

Lemma 1. Every tree-decomposition of a vertex-weighted graph has a bag that is a half-separator of the graph. Such a bag is called center of the treedecomposition. 
For the sake of presentation, we prove the following folklore results.

\section{Proposition 1}

1. Every weighted treewidth-t graph is strongly $\lceil(t+1) / 2\rceil$-path separable.

2. Every weighted planar graph is strongly 3-path separable.

3. Every weighted $n$-vertex graph is strongly $\lceil n / 4\rceil$-path separable

4. The uniform 2 weighted clique $K_{4 k+1}$ is not $k$-path separable.

Proof.

1. Consider any subgraph $H$ of a weighted graph $G$. The treewidth of $H$ is at most the treewidth of $G$. So $H$ has a tree-decomposition of width $\leqslant t$. By Lemma 1 the center $C$ of the tree-decomposition is a half-separator. It can be covered by at most $\lceil|C| / 2\rceil \leqslant\lceil(t+1) / 2\rceil$ shortest paths. Therefore, $H$ has a strong $\lceil(t+1) / 2\rceil$-path separator, and thus $G$ is strongly $\lceil(t+1) / 2\rceil$-path separable.

2. It is well-known that every planar graph has a tree-decomposition such that every bag consists of at most three shortest paths. This comes from the well-known fact that every planar graph having a depth- $h$ rooted tree has a tree-decomposition where each bag consists of 3 paths of the tree starting from the root (see [5][pp. 305]). By Lemma 1] the center $C$ of the tree-decomposition is a half-separator. So, $C$ forms a strong 3 -path separator.

3. Consider any subgraph $H$ of a weighted graph $(G, \omega)$ with $n$ vertices. Let $W$ be the smallest set of vertices in $H$ such that $\omega(W) \geqslant \omega(H) / 2$. Thus, the components of $H \backslash W$ have weight $\leqslant \omega(H) / 2$. It is clear that $W$ contains at most half the vertices of $H$, i.e., $|W| \leqslant\lceil|V(H)| / 2\rceil$. A set of at most $\lceil|W| / 2\rceil$ shortest paths suffices to cover $W$. Therefore, $H$ has a strong $k$-path separator with $k=\lceil\lceil|V(H)| / 2\rceil / 2\rceil \leqslant\lceil n / 4\rceil$, completing the proof of Point 3 .

4. Let us show that the uniform weighted $K_{4 k+1}$ has no $k$-path separator. Indeed, every $k$-path separator $S$ consists of at most $2 k$ vertices since every shortest path in a clique consists of an edge. $K_{4 k+1} \backslash S$ is a clique on at least $2 k+1$ vertices, so $S$ is not a half-separator.

As remark in 11, the $k$-path separability of minor-free graphs holds also for vertex-weighted graphs. However the formal proof of this result cannot be considered as folklore, and its self-contained proof is currently more than the page limitation of this paper.

One the of unresolved problem we left open is to know whether they are planar graphs that are not 2-path separable.

\section{Face-Separable Graphs}

As we will see later in Section 4 , graphs that are $k$-path separable have strong structural properties. In particular, planarity plays an important role, at least for $k=1$ in the light of Theorem 3. In this section we will see that, a half-separator

\footnotetext{
${ }^{2}$ That is with a unit weight for all vertices and edges.
} 
of a special "shape" implies a low path separability of the graph. Moreover, this half-separator is defined independently of the shortest path metric of the graph, it only depends on the vertex-weight function.

A half-separator $S$ of a weighted graph $G$ is a face-separator if $G$ has a plane embedding such that $S$ is the border of a face. A graph is face-separable if every induced subgraph has a face-separator.

By definition, outerplanar graphs are face-separable, since the outerface contains all vertices of the graph. We will see that the family of face-separable graphs includes more general graphs, like the series-parallel graphs, the subdivisions of a $K_{4}$ (Proposition 2), and even includes some unbounded treewidth planar graphs (Proposition 3).

The main result of this section is:

Theorem 1. Every face-separable weighted graph is strongly 2-path separable.

The bound given by Theorem 1 is best possible because there are face-separable graphs that are not 1-path separable. This can be proved by combining Proposition 2 and the fact there are treewidth-2 graphs and subdivisions of $K_{4}$ that are not 1-path separable - see Fig. 3 .

Proposition 2. Every weighted treewidth-2 graph or weighted subdivision of $K_{4}$ is face-separable.

Proof. Let $(G, \omega)$ be any weighted treewidth- 2 graph. It is known that every treewidth-2 graph is a subgraph of a series-parallel graph, and in particular a planar graph. As any subgraph of $G$ is also a treewidth-2 graph, it is sufficient to prove that $G$ has a face-separable. We consider the graph $H$ obtained from $G$ by adding as many edges as possible while preserving a treewidth- 2 graph. Let $T$ be a tree-decomposition of $H$ of width 2 , and let $C$ be the center of $T$. Bag $C$ is composed of a $K_{3}$. We embed $H$ in the plane such that $C$ is the border of a face of this embedding. This is possible by moving some subgraph from inside the $K_{3}$ to outside. If not, $H$ would contain a $K_{4}$-minor, contradicting the fact that $H$ has treewidth 2 . We can now remove the edges that have been added to $H$ in order to obtain $G$, and we consider the border $S$ of the face containing the three vertices of $C$. Such a face exists since deleting edges can only enlarge the existing faces of a plane embedding. We have $S \subseteq C$, and $C$ is a half-separator of $H$ (Lemma 1). Note that $H$ has the same total weight of $G$ (we have added only edges). It follows that $S$ is a half-separator for $G$. This completes the first part of the statement of the proposition.

Consider now a subdivision $G$ of $K_{4}$ having a vertex-weight function $\omega$, and $H$ be an induced subgraph of $G$. If $H$ is a proper subgraph of $G$ (i.e., $H \neq G$ ), then $H$ is outerplanar and thus has a face-separator. So, assume that $H=G$.

We assume given a plane embedding of $H$. We denote by $v_{1}, \ldots, v_{4}$ the four degree-3 vertices of $H$, and by $P_{i, j}$ the path between $v_{i}$ and $v_{j}$, for all $i, j \in$ $\{1, \ldots, 4\}$. Let $w_{i}=\omega\left(v_{i}\right)$, and let $p_{i, j}=\omega\left(P_{i, j} \backslash\left\{v_{i}, v_{j}\right\}\right)$ be the sum of the weights of vertices on $P_{i, j}$ excluding its extremities. 
Let assume that $H$ has no face-separator. There are four possible faces $F_{1}, \ldots, F_{4}$, each one bordered by three paths. Faces are ordered such that whenever the border of $F_{i}$ is removed, the remaining component is composed of three paths sharing vertex $v_{i}$. The total weight of this component is $w_{i}+\sum_{j \neq i} p_{i, j}$. As the border of $F_{i}$ is not a face-separator, we have $w_{i}+\sum_{j \neq i} p_{i, j}>\omega(H) / 2$. This holds for each $i \in\{1, \ldots, 4\}$. Summing these four equations, it turns out (observe that each path $p_{i, j}$ occurs twice in this sum):

$$
\sum_{i} w_{i}+2 \sum_{i \neq j} p_{i, j}>2 \omega(H)=2\left(\sum_{i} w_{i}+\sum_{i \neq j} p_{i, j}\right) .
$$

It implies $0>\sum_{i} w_{i}$ : a contradiction, by definition $\omega(v) \geqslant 0$ for each vertex $v$. Therefore, one of the face $F_{i}$ is a face-separator for $H$, that completes the proof.

Up to now, the graphs we have proved to be face-separable are all of treewidth $\leqslant 3$. From Proposition 1 (Point 1), they are 2-path separable. It is natural to ask whether all face-separable graphs have such a low treewidth property. We answer negatively to this question.

Proposition 3. For every $n$, there is a uniform weighted face-separable graph with at most $n$ vertices whose treewidth is $\Omega(\log \log n)$.

Proof. (Sketch). The proof is based on the construction of a graph called $G_{p}$, for integral $p \geqslant 1$. It has treewidth at least $k=p-O(\log \log p)$ because we can show it contains a $k \times k$-grid minor, and the number of vertices of $G_{p}$ is $n<2^{2^{p}}$. In other words, the treewidth of $G_{p}$ is at least $\log \log n-O\left(\log ^{(4)} n\right)$.

Graph $G_{p}$ is composed of a tree $T_{p}$ of depth $p$ where each vertex of depth $i<p$ has exactly $d(i)$ children, for some function $d$ defined later. Furthermore, for each depth $i$, a path linking all depth- $i$ vertices is added to $T_{p}$ to form $G_{p}$. Let us denote by $L(i)$ the number of depth- $i$ vertices in $T_{p}$. The values $L(i)$ and $d(i)$ obey to the following induction: $L(0)=1$ and $L(i)=L(i-1) \cdot d(i-1)$, where $d(i)=\sum_{j=0}^{i} L(j)$. The first values of $L(i)$ and $d(i)$ are given in the table hereafter, and $G_{4}$ is depicted on Fig. 1 .

\begin{tabular}{c|ccccccc}
$i$ & 0 & 1 & 2 & 3 & 4 & 5 & $\ldots$ \\
\hline$L$ & 1 & 1 & 2 & 8 & 96 & 10368 & $\ldots$ \\
$d$ & 1 & 2 & 4 & 12 & 108 & 10464 & $\ldots$
\end{tabular}

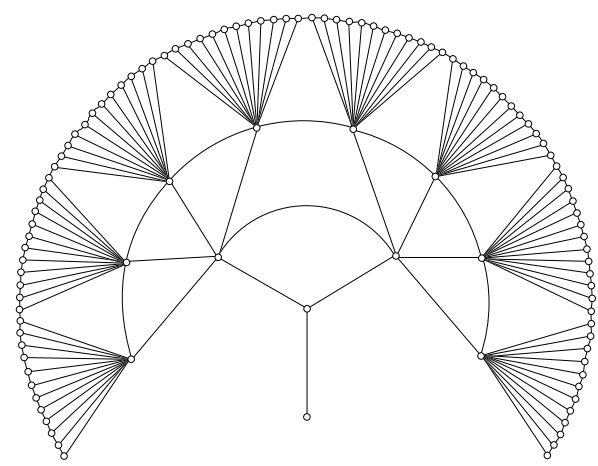

Fig. 1. The graph $G_{4}$ with 108 vertices 
To prove Proposition 3. we show that every subgraph $H$ of $G_{p}$ contains a face-separator. An important property we use is that in $G_{p}$, the number $d(i)$ of children for a vertex of depth $i$ is at least the vertex number of the graph induced by $T_{i-1}$. The key point is that $H$ is either outerplanar, or there must exist a vertex $v$ of depth $i$ in $T_{p}$ such that all its children belongs to the border of the outerface of $H$. In the first case, $H$ is trivially face-separable. In the second one, and using the property on $d(i)$, we derive that at least half the vertices of $H$ lie on the outerface.

\section{The Hierarchy of Separable Graphs}

For every integer $k \geqslant 1$, we denote by $\mathrm{PS}_{k}$ the family of all the graphs $G$ that are $k$-path separable for every weight function $\omega$. More formally,

$$
\mathrm{PS}_{k}=\{G \mid \forall \omega,(G, \omega) \text { is } k \text {-path separable }\} .
$$

We define similarly the family $\mathrm{SPS}_{k}$ of all the graphs that are strongly $k$-path separable for every weight function.

We have seen that every weighted planar graph is strongly 3 -path separable. In other words, planar graphs are in $\mathrm{SPS}_{3}$. In Section 3 , we have seen that every subdivided outerplanar graph is face-separable, and thus strongly 2-path separable. Thus this family is in $\mathrm{SPS}_{2}$. We will show in Proposition 6 that outerplanar graphs are in actually in $\mathrm{PS}_{1}$. Obviously, families $\mathrm{PS}_{1}$ and $\mathrm{SPS}_{1}$ coincide.

Clearly, for each $k, \mathrm{SPS}_{k} \subset \mathrm{PS}_{k}$ since a strongly $k$-path separator is a particular $k$-path separator. Also, the hierarchies $\mathrm{PS}_{1} \subset \cdots \subset \mathrm{PS}_{k}$ and $\mathrm{SPS}_{1} \subset$ $\cdots \subset \mathrm{SPS}_{k}$ are strict because of the complete graph. By Proposition 1 (points 3 and 4$), K_{4 k+1} \in \mathrm{SPS}_{k+1}$ and $K_{4 k+1} \notin \mathrm{PS}_{k}$. The family $\mathrm{PS}_{k}$ is however much larger than $\mathrm{SPS}_{k}$ as suggested by the next proposition.

Proposition 4. For each $k>4$, there is a graph $G_{k}$ with $O\left(k^{2}\right)$ vertices such that $G_{k} \notin \mathrm{SPS}_{k}$, but $G_{k} \in \mathrm{PS}_{4}$.

Proof. Consider the graph $G_{k}$ composed of a $2(k+1) \times 2(k+1)$-grid in which a vertex $v$ is connected to all the vertices of the grid. $G_{k}$ has $4 k^{2}+5$ vertices. Vertex and edge weights are unitary. As shown in [1, $G_{k}$ has no strong $k$-path separator, the removal of any set of $k$ shortest paths deletes at most $2 k+1$ vertices, and $2(k+1)$ are required to halve the graph. Therefore, $G_{k} \notin \mathrm{SPS}_{k}$. However, for every weight function $\omega,\left(G_{k}, \omega\right)$ has a 4 -path separator. The first path consists of the universal vertex $v$, and the three others are defined as in the planar case (since $G_{k} \backslash\{v\}$ is planar, and thus 3-path separable). Therefore, $G_{k} \in \mathrm{PS}_{4}$.

For the study of $\mathrm{PS}_{k}$ and $\mathrm{SPS}_{k}$ graphs families, the next proposition tell us that we can always assume that graphs are biconnected.

Proposition 5. A graph belongs to $\mathrm{PS}_{k}$ (reps. $\mathrm{SPS}_{k}$ ) if and only if all its bicomponents belong to $\mathrm{PS}_{k}$ (resp. SPS S. . 


\subsection{Closed under Minor Taking}

The remarkable property of $\mathrm{PS}_{k}$ and $\mathrm{SPS}_{k}$ families is they are closed under minor taking. From the Graph Minor Theorem, such families can be characterized by a finite list of forbidden minors, and membership of a given graph in one of these families can be done in time $O\left(n^{3}\right)$ for fixed $k$.

Theorem 2. For each integer $k \geqslant 1$, the families $\mathrm{PS}_{k}$ and $\mathrm{SPS}_{k}$ are minorclosed.

Proof. We give the proof for the family $\mathrm{SPS}_{k}$, the proof for $\mathrm{PS}_{k}$ is similar. Let $H$ be any minor of a graph $G$. We will prove that if $G$ is $k$-path separable, then $H$ is $k$-path separable too. To prove that $H$ is $k$-path separable, we need to prove the property for every induced subgraph of $H$. However, since every subgraph of $H$ is also a minor of $G$, we simply show that $H$ has a $k$-path separator.

It is not difficult to see that if $H$ is a minor of $G$, then with each vertex $u$ of $H$ we can associate a connected subgraph of $G$, called super-node of $u$, such that if $(u, v)$ is an edge of $H$, then there exists an edge of $G$, called super-edge of $(u, v)$, connecting a vertex of the super-node of $u$ and a vertex of the super-node of $v$. (If there are several such edges we select only one.) The super-nodes must be pairwise disjoint (see Fig. 21).

Let $\omega_{H}$ be any weight function on $H$. From $\omega_{H}$, we construct a weight function $\omega_{G}$ on $G$ as follows. For every edge $(x, y)$ of $G$ that is a super-edge of $(u, v)$ (colored black on Fig. 2), we set $\omega_{G}(x, y)=\omega_{H}(u, v)$. For every edge $(x, y)$ of $G$ such that $x$ and $y$ both belongs to the same super-node (called internal-edge and dashed on Fig. (2), we set $\omega_{G}(x, y)=0$. And, for all other edges $(x, y)$ of $G$ (called external-edge and colored red on Fig. (2), we set $\omega_{G}(x, y)=1+\sum_{e \in E(H)} \omega_{H}(e)$, so that the cost of a path in $G$ using any such edge is strictly larger than the cost of any simple path in $H$. The weight of a vertex $x$ that belongs to the super-node of $u$ is $\omega_{G}(x)=\omega_{H}(u) / t_{u}$, where $t_{u}$ is the number of vertices of the super-node of
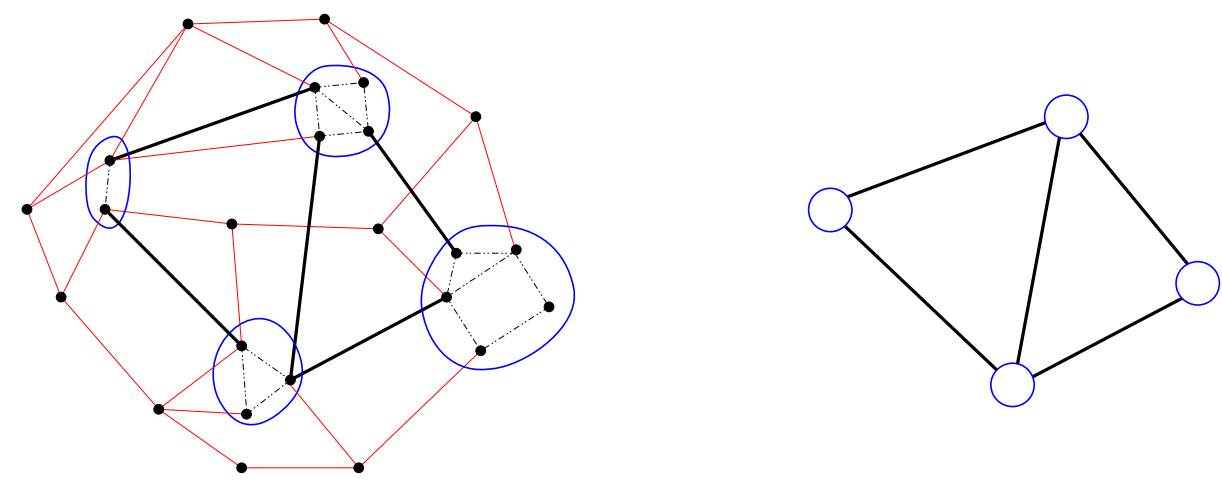

Fig. 2. A graph $G$ and a minor $H$ 
$u$. Note that the sum of weights of the vertices of the super-node of $u$ is precisely $\omega_{H}(u)$. The weight of all other vertices is 0 . Observe that $\omega_{G}(G)=\omega_{H}(H)$.

Since $G \in \operatorname{SPS}_{k}$, the weighted graph $\left(G, \omega_{G}\right)$ has a $k$-path separator $S_{G}$ consisting of $k$ shortest paths in $G$. Let $H_{0}, H_{1}, \ldots$ be the components of $H$, and assume that $\omega_{H}\left(H_{0}\right)$ is maximum. With each path $P$ of $S_{G}$ that intersects a super-node of a vertex of $H_{0}$, we associate a path $Q$ in $H_{0}$ as follows. Let $\left(U_{0}, \ldots, U_{t}\right)$ be the ordered sequence of all the super-nodes of vertices of $H_{0}$ traversed by $P$. We denote by $u_{i}$ the vertex of $H_{0}$ such $U_{i}$ is the super-node of $u_{i}$. Path $Q$ is obtained by adding an edge between $u_{i-1}$ to $u_{i}$, for each $i \in\{1, \ldots, t\}$. We claim that the set composed of each path $Q$ constructed from $P$ as above, and denote by $S_{H}$, is a half-separator of $H$.

First, let us show that $Q$ is a shortest path in $H_{0}$ (and thus in $H$ ). Path $P$ between the last vertex of $U_{i-1}$ and the first vertex of $U_{i}$ consists of the superedge of $\left(u_{i-1}, u_{i}\right)$, because $H_{0}$ is connected and the weight of this super-edge is less than the weight of any external-edges. Thus $Q$ is a path in $H_{0}$. Now, assume that there exists a path $Q^{\prime}$ in $H_{0}$, from $u_{0}$ to $u_{t}$, that is shorter than $Q$. Then, from $Q^{\prime}$ we can construct a shorter path in $G$ (shorter than $P$ ) from the last vertex of $U_{0}$ to the first vertex of $U_{t}$. This is due to the fact that each super-node is connected and internal-edges have weight 0 . This contradicts that $P$ is a shortest path, hence $Q$ is a shortest path in $H_{0}$.

It remains to show that $S_{H}$ is a half-separator of $H$. Observe that for $i \neq 0$, $\omega_{H}\left(H_{i}\right) \leqslant \omega_{H}(H) / 2$ because $\omega\left(H_{0}\right)$ is maximum. Let $X_{H_{0}}$ be the set of vertices of any component in $H_{0} \backslash S_{H}$. Then, there must exists a component $X_{G}$ in $G \backslash S_{G}$ wholly containing all the super-nodes of the vertices of $X_{H_{0}}$. Let $v$ be a vertex of $X_{H_{0}}$ whose its super-node belongs to none component of $G \backslash S_{G}$. Then, there exists a vertex of this super-node that is in $S_{G}$. From our construction, $v$ belongs to $S_{H}$ (vertices of $Q$ and super-nodes of $P$ correspond): contradiction. Therefore, $\omega_{H}\left(X_{H_{0}}\right) \leqslant \omega_{G}\left(X_{G}\right)$. Moreover, $\omega_{G}\left(X_{G}\right) \leqslant \omega_{G}(G) / 2=\omega_{H}(H) / 2$. Thus, $S_{H}$ is a half-separator of $H$, completing the proof.

\subsection{One-Path Separable Graphs}

In this part, we concentrate our attention to the graphs that belong to $\mathrm{PS}_{1}$. We have seen in Proposition 2, that the subdivisions of outerplanar graph or of $K_{4}$ are face-separable, and thus belong to $\mathrm{PS}_{2}$ (and even to $\mathrm{SPS}_{2}$ ). Actually, outerplanar graphs are in $\mathrm{PS}_{1}$ :

Proposition 6. Every weighted outerplanar graph is 1-path separable.

Unfortunately, Proposition [6] does not generalize to treewidth-2 graphs. As depicted on Fig. 3, there are simple series-parallel graphs and subdivisions of $K_{4}$ that are not in $\mathrm{PS}_{1}$.

The family $\mathrm{PS}_{1}$ does not reduce to outerplanar graphs, as shown in Proposition [7. A globe graph is a subdivision of $K_{2, r}$, for some $r$, in which the two degree- $r$ vertices may be adjacent.

Proposition 7. Every weighted globe graph is 1-path separable. 

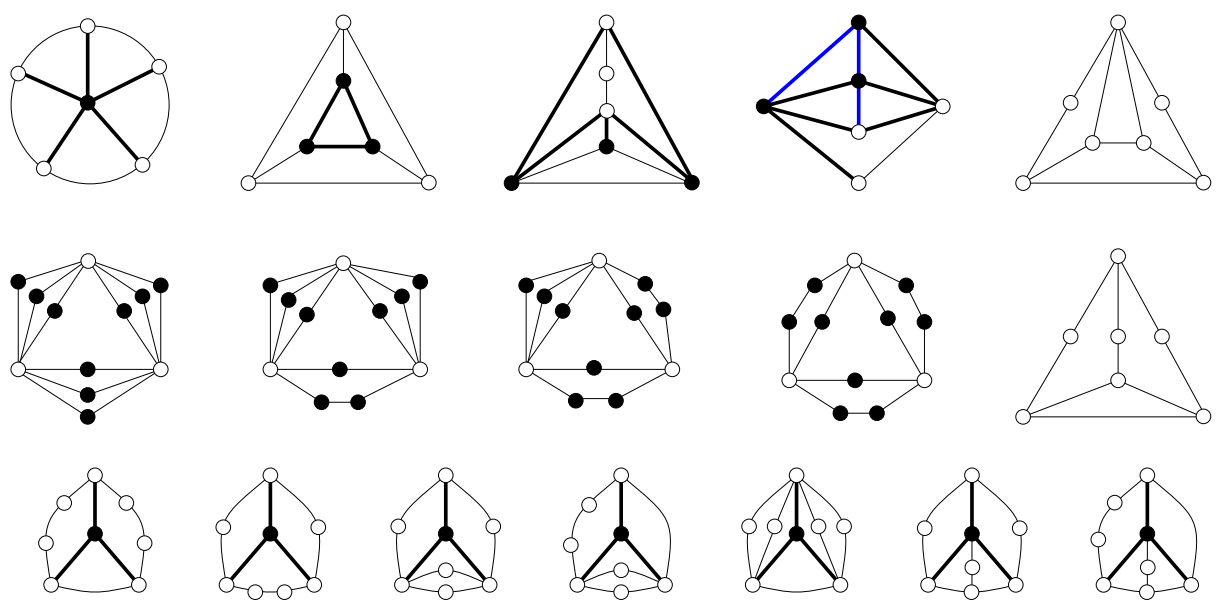

Fig. 3. Forbidden minors for planar graphs in $\mathrm{PS}_{1}$. Black vertices and bold edges have weight 2 , blue edges have weight 3 , other vertices and edges have weight 1 . The two non-planar forbidden minors are $K_{5}$, and a $K_{3,3}$ whose one edge is subdivided into two edges.

A first attempt to characterize $\mathrm{PS}_{1}$ is given by Theorem 3

Theorem 3. Every biconnected graph of $\mathrm{PS}_{1}$ is either isomorphic to $K_{3,3}$, or planar and excludes the list of minors depicted on Fig. 3 .

Proof. Let $(G, \omega)$ be a biconnected weighted graph with $G \in \mathrm{PS}_{1}$. First assume that $G$ is not planar. From Kuratowski's criteria, $G$ contains a subdivision of $K_{5}$ or $K_{3,3}$.

The complete graph $K_{5}$ is not 1-path separable graph from Proposition 1 (cf. Point 4 with $k=2$ ). From Theorem 2 , it follows that $G$ cannot contain a subdivision of $K_{5}$, so it must contain a subdivision of $K_{3,3}$.

We shall proof that a subdivision of $K_{3,3}$ in which only one edge is subdivided into two edges is not 1-path separable. Denote by $M$ this graph, and set unitary all the weights so that the total vertex-weight is 7 . The diameter of $M$ is two. The deletion of any shortest path deletes at most three vertices. Moreover, such a deletion cannot disconnect $M$, and thus leaves a component with at least $4>7 / 2$ vertices. $M$ is not 1-path separable. Therefore, if $G$ is not planar, then $G$ can only be isomorphic to $K_{3,3}$.

We prove now that $K_{3,3}$ is 1 -path separable. Denote by $\left\{x_{1}, x_{2}, x_{3}\right\}$ and $\left\{y_{1}, y_{2}, y_{3}\right\}$ the vertex set of each part of $K_{3,3}$. We first show that $K_{3,3}$ has a 1-path separator. Later we will prove it for all its induced subgraphs. W.l.o.g. assume that $\omega\left(x_{1}\right) \geqslant \omega\left(x_{2}\right) \geqslant \omega\left(x_{3}\right)$.

Define $P_{1}$ be a shortest path from $x_{1}$ to $x_{2}$, and assume $P_{1}$ contains $y_{i_{1}}$. Define $P_{2}$ be a shortest path from $y_{i_{2}}$ to $y_{i_{3}}$ (with $i_{1}, i_{2}, i_{3}$ pairwise different indices), and assume it contains $x_{j_{1}}$ (denote by $j_{2}, j_{3}$ the two other $x$ 's indices). We show that $P_{1}$ or $P_{2}$ is a 1-path separator. By contradiction, if $P_{1}$ is not a half-separator, 
then (and similarly for $\left.P_{2}\right) \omega\left(P_{1}\right)<\omega\left(K_{3,3}\right) / 2$ and $\omega\left(G \backslash P_{1}\right)>\omega\left(K_{3,3}\right) / 2$. As $\omega\left(P_{1}\right)$ is lower bounded by $\omega\left(x_{1}\right)+\omega\left(x_{2}\right)+\omega\left(y_{i_{1}}\right)$ and $\omega\left(G \backslash P_{1}\right)$ upper bounded by $\omega\left(x_{3}\right)+\omega\left(y_{i_{2}}\right)+\omega\left(y_{i_{3}}\right)$ (and similarly for $\left.P_{2}\right)$, it follows that:

$$
\begin{aligned}
\omega\left(x_{1}\right)+\omega\left(x_{2}\right)+\omega\left(y_{i_{1}}\right) & <\omega\left(x_{3}\right)+\omega\left(y_{i_{2}}\right)+\omega\left(y_{i_{3}}\right) \\
\omega\left(x_{j_{1}}\right)+\omega\left(y_{i_{2}}\right)+\omega\left(y_{i_{3}}\right) & <\omega\left(x_{j_{2}}\right)+\omega\left(x_{j_{3}}\right)+\omega\left(y_{i_{1}}\right)
\end{aligned}
$$

By summing these equations, we obtain:

$$
\begin{aligned}
\omega\left(x_{1}\right)+\omega\left(x_{2}\right)+\omega\left(x_{j_{1}}\right) & <\omega\left(x_{3}\right)+\omega\left(x_{j_{2}}\right)+\omega\left(x_{j_{3}}\right) \\
\Rightarrow \quad \omega\left(x_{1}\right)+\omega\left(x_{2}\right)+\omega\left(x_{3}\right) & <\omega\left(x_{3}\right)+\omega\left(x_{j_{2}}\right)+\omega\left(x_{j_{3}}\right) \leqslant \omega\left(x_{3}\right)+\omega\left(x_{2}\right)+\omega\left(x_{1}\right)
\end{aligned}
$$

since, by assumption, $\omega\left(x_{3}\right) \leqslant \omega\left(x_{j_{1}}\right)$ and $\omega\left(x_{j_{2}}\right)+\omega\left(x_{j_{3}}\right) \leqslant \omega\left(x_{2}\right)+\omega\left(x_{1}\right)$. This leads to a contradiction. Thus, $P_{1}$ or $P_{2}$ is a 1 -path separator for $K_{3,3}$.

Now, let $H$ be any induced subgraph of $K_{3,3}$. We use similar notations excepted that the two vertex-sets are $\left\{x_{1}, \ldots, x_{p}\right\}$ and $\left\{y_{1}, \ldots, y_{q}\right\}$ with $1 \leqslant p \leqslant$ $q \leqslant 3$. If $p+q \leqslant 4$, then $H$ is outerplanar, and thus 1-path separable by Proposition 6. We are left with the case $p=2$ and $q=3$, the case $p=q=3$ is already done. We define similarly the two paths $P_{1}$ and $P_{2}$, i.e., a shortest path from $x_{1}$ to $x_{2}$ containing $y_{i_{1}}$, and the shortest path between $y$ 's vertices different from $y_{i_{1}}$ and through $x_{j_{1}}$. If these both paths are not half-separators, then Eq. (1) and (2) rewrite in (vertices $x_{3}$ and $x_{j_{3}}$ do not exist anymore):

$$
\begin{aligned}
\omega\left(x_{1}\right)+\omega\left(x_{2}\right)+\omega\left(y_{i_{1}}\right) & <\omega\left(y_{i_{2}}\right)+\omega\left(y_{i_{3}}\right) \\
\omega\left(x_{j_{1}}\right)+\omega\left(y_{i_{2}}\right)+\omega\left(y_{i_{3}}\right) & <\omega\left(x_{j_{2}}\right)+\omega\left(y_{i_{1}}\right)
\end{aligned}
$$

Summing these equations, we obtain:

$$
\omega\left(x_{1}\right)+\omega\left(x_{2}\right)+\omega\left(x_{j_{1}}\right)<\omega\left(x_{j_{2}}\right),
$$

a contradiction since vertex-weights are non-negative and $\omega\left(x_{j_{2}}\right) \leqslant \omega\left(x_{1}\right)$. Thus, $P_{1}$ or $P_{2}$ is a 1-path separator for $H$.

Therefore, we have proved that the only non-planar graph of $\mathrm{PS}_{1}$ is $K_{3,3}$. For planar graphs, we manage to find forbidden minors represented in Fig. 3 . To prove that each minor $M$ of this list is indeed excluded, we exhibit a particular weight function $\omega$ for $M$. Actually, each vertex and edge has weight 1 or 2 as depicted on Fig. 3. We then exhaustively check that, for each pair $u, v$ of vertices of $M$, the deletion of any shortest path from $u$ to $v$ leaves a component of weight $>\omega(M) / 2$.

To illustrate this, consider for instance the "wheel graph", composed of a cycle of length 5 and a degree- 5 vertex, called hereafter center, connected of all vertices of the cycle. The total weight of the graph is 7 , the center has weight 2. Any shortest path from the center to a non-center vertex consists of one edge. Therefore its deletion leaves a path of 4 vertices, so of weight 4 . Any shortest path between two non-center vertices consists of 2 edges at most, so leaving a component with the center and two (or more) non-center vertices, thus of weight at least 4 . In both cases, the weight is $>7 / 2$. This graph has no half-separator composed of a shortest path, and thus is not in $\mathrm{PS}_{1}$. 


\section{Conclusion}

In this paper we have investigated the family of graphs that are $k$-path separable. Graph Minor Theory implies that such a family can be characterized by a finite set of forbidden minors that we have started to list for $k=1$.

We propose here a list of further researches.

1. Determine the full list of forbidden minors for $k$-path separable graphs and for $k=1$.

2. Find an explicit linear time algorithm to determine if a graph is $k$-path separable, for fixed $k$.

3. Prove or disprove that planar graphs are 2-path separable.

4. Prove NP-completeness for the problem of determining whether a given weighted graph has a $k$-path separator.

5. Extend the study to more general isometric separators, not only shortest paths.

\section{References}

1. Abraham, I., Gavoille, C.: Object location using path separators. In: $25^{\text {th }}$ Annual ACM Symp. on Principles of Distributed Comp. (PODC), pp. 188-197 (2006)

2. Abu-Khzam, F.N., Collins, R.L., Fellows, M.R., Langston, M.A., Suters, W.H., Symons, C.T.: Kernelization algorithms for the vertex cover problem: Theory and experiments. In: $6^{\text {th }}$ Workshop ALENEX, pp. 62-69 (2004)

3. Chepoi, V.D., Dragan, F.F., Estellon, B., Habib, M., Vaxès, Y.: Diameters, centers, and approximating trees of delta-hyperbolic geodesic spaces and graphs. In: $24^{\text {st }}$ Annual ACM Symposium on Computational Geometry (SoCG), pp. 59-68 (2008)

4. Dourisboure, Y., Gavoille, C.: Tree-decompositions with bags of small diameter. Discrete Mathematics 307(16), 2008-2029 (2007)

5. Flum, J., Grohe, M.: Parametrized Complexity Theory. Springer, Heidelberg (2006)

6. Koster, A.M.C.A., Bodlaender, H.L., van Hoesel, S.P.M.: Treewidth: Computational experiments. In: Elsevier (ed.) $1^{\text {st }}$ Cologne-Twente Workshop on Graphs and Combinatorial Optimization, vol. 8, pp. 54-57. ENDM, Amsterdam (2001)

7. Lipton, R.J., Tarjan, R.E.: A separator theorem for planar graphs. SIAM Journal on Applied Mathematics 36(2), 177-189 (1979)

8. Robertson, N., Seymour, P.D.: Graph minors. XIII. The disjoint paths problem. Journal of Combinatorial Theory, Series B 63(1), 65-110 (1995)

9. Robertson, N., Seymour, P.D.: Graph minors. XVI. Excluding a non-planar graph. Journal of Combinatorial Theory, Series B 89(1), 43-76 (2003)

10. Robertson, N., Seymour, P.D.: Graph minors. XX. Wagner's conjecture. Journal of Combinatorial Theory, Series B 92(2), 325-357 (2004)

11. Thorup, M.: Compact oracles for reachability and approximate distances in planar digraphs. Journal of the ACM 51(6), 993-1024 (2004)

12. Umezawa, K., Yamazaki, K.: Tree-length equals branch-length. Discrete Mathematics 309(13), 4656-4660 (2009) 